\title{
Laser Induced Quadrupole-Dipole Collisional Energy Transfer in $\mathrm{Ca}-\mathrm{Sr}$
}

\author{
Z.Z. LU*, Y.L. Sun, L. MA AND J.F. LiU \\ School of Physics and Optoelectronic Engineering, Xidian University, Xi'an 710071, China
}

(Received February 4, 2015; in final form April 24, 2015)

\begin{abstract}
A laser induced quadrupole-dipole collisional energy transfer system of $\mathrm{Ca}-\mathrm{Sr}$ is proposed and the four-state quadrupole-dipole laser induced collisional energy transfer model is presented. The excitation spectrum calculated for the $\mathrm{Ca}-\mathrm{Sr}$ system is characterized by a doublet structure and a width which is about twice wider than the dipole-dipole laser induced collisional energy transfer results. Calculating results show that the full width at half peak of the profile becomes larger and the quasi-static wing tends to be a broad flat as the field intensity increases, which are different from the reported results of dipole-dipole laser induced collisional energy transfer processes, dipole-quadrupole and quadrupole-quadrupole. A cross-section of $2.48 \times 10^{-14} \mathrm{~cm}^{2}$ at a laser field intensity of $5.21 \times 10^{7} \mathrm{~V} / \mathrm{m}$ is obtained which indicates that the quadrupole-dipole process can be an effective way to transfer energy selectively.
\end{abstract}

DOI: $10.12693 /$ APhysPolA.127.1620

PACS: $32.70 . \mathrm{Jz}, 34.50 .-\mathrm{S}$

\section{Introduction}

Laser-induced collisional energy transfer (LICET) is an optical process in which a laser field is applied during the collision between two different particles to induce energy transfer from a level of one atomic species to a level of another atomic species. It cannot occur unless the two mechanisms, i.e., a collision and a radiative interaction, are both present. Neither of the two mechanisms can singly induce this interparticle transition. For many years, LICET has been of considerable interest [1-18], because the cross-section of interparticle inelastic collisions can be greatly increased by the joint action of the collisional and radiative interactions. In addition, the ability to excite the special target level of the chosen particle permits potential applications of LICET in controlling pathways of chemical reactions, thus making a detailed understanding of atomic dynamical processes possible.

As plenty of the experimental studies have been made [1-6], people also have proposed many theoretical models on LICET [7-15]. Among these models, it was the two-level model established by Harris et al. that influenced most [9]; later this two-level approximation was generalized by Agresti et al. into three-level perturbative theory [11].

The previous experimental and theoretical researches on the LICET were mainly concentrated on the dipoledipole process in which one atom made a parity-allowed transition while another made a parity-nonallowed transition. In this paper, a laser induced quadrupole-dipole collisional energy transfer system of $\mathrm{Ca}-\mathrm{Sr}$ in which both species made parity-nonallowed transitions is proposed and a laser induced quadrupole-dipole LICET four-level model is presented. Based on our theory results, we

${ }^{*}$ corresponding author; e-mail: luhit@126.com can clearly see that the laser induced quadrupole-dipole collisional energy transfer process is much faster than the reported dipole-dipole $[1,9,10,15]$ and dipolequadrupole [17] collisional process. This $\mathrm{Ca}-\mathrm{Sr}$ system is characterized by a new type of compound system energy level shift caused by the joint action of the collisional and radiative interactions.

\section{Theory}

A diagram of the relevant energy levels of the $\mathrm{Ca}-\mathrm{Sr}$ system involved in the LICET process is shown in Fig. 1.
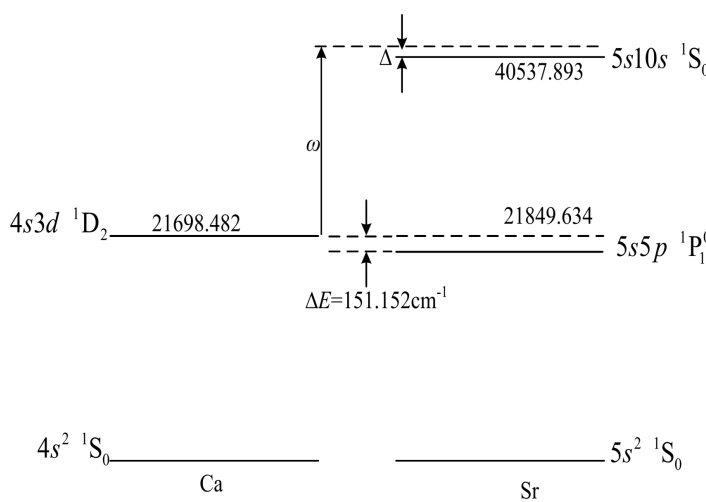

Fig. 1. The LICET transition in $\mathrm{Ca}-\mathrm{Sr}$ system. The energy levels in the figure are given in $\mathrm{cm}^{-1}$.

During the collision between excited $\mathrm{Ca}\left(4 s 3 d^{1} D_{2}\right)$ and $\operatorname{Sr}\left(5 s 2^{1} S_{0}\right)$ atoms, the applied transfer laser field induces a transition of $\mathrm{Sr}$ from the ground state to a virtual level. The quadrupole-dipole coupling then de-excites the $\mathrm{Ca}$ atom to the ground state while it excites the Sr atom from the virtual level to the $\operatorname{Sr}\left(5 s 10 s^{1} S_{0}\right)$ target state. The process can be expressed by the following equation:

$$
\begin{aligned}
& \mathrm{Ca}\left(4 s 3 d^{1} D_{2}\right)+\operatorname{Sr}\left(5 s^{2}{ }^{1} S_{0}\right) \\
& +\hbar \omega(530.802 \mathrm{~nm}) \longrightarrow \mathrm{Ca}\left(4 s^{2}{ }^{1} S_{0}\right)+\operatorname{Sr}\left(5 s 10 s^{1} S_{0}\right) .
\end{aligned}
$$


In our calculation, the multipole collisional system is regarded to be a quasi-molecular system. During the collision process, there are four important compound states (see Eq. (1)). $|2\rangle$ is the initial state that the atom $\mathrm{Ca}$ is in the excited state of $\mathrm{Ca}\left(4 s 3 d^{1} D_{2}\right)$ and the atom $\mathrm{Sr}$ is in the ground state. The $|4\rangle$ is the final state that the atom $\mathrm{Ca}$ is in the ground state and the atom $\mathrm{Sr}$ is in the excited state of $\operatorname{Sr}\left(5 s 10 s^{1} S_{0}\right) .|1\rangle$ and $|3\rangle$ are two important intermediate states that have contribution to the final state transition.

$$
\left.\begin{array}{l}
|1\rangle=\left|\mathrm{Ca}\left(4 s^{2}{ }^{1} S_{0}\right)\right\rangle\left|\operatorname{Sr}\left(5 s 5 p^{1} P_{1}^{0}\right)\right\rangle, \\
|2\rangle=\left|\mathrm{Ca}\left(4 s 3 d^{1} D_{2}\right)\right\rangle\left|\operatorname{Sr}\left(5 s^{2}{ }^{1} S_{0}\right)\right\rangle, \\
|3\rangle=\left|\mathrm{Ca}\left(4 s 3 d^{1} D_{2}\right)\right\rangle\left|\operatorname{Sr}\left(5 s 5 p^{1} P_{1}^{0}\right)\right\rangle, \\
|4\rangle=\left|\mathrm{Ca}\left(4 s^{2}{ }^{1} S_{0}\right)\right\rangle\left|\operatorname{Sr}\left(5 s 10 s^{1} S_{0}\right)\right\rangle .
\end{array}\right\}
$$

The wave function of the coupling system can be expressed by the four compound states

$$
\Psi=\sum_{n=1}^{4} c_{n}(t)|n\rangle \exp \left(-\mathrm{i} E_{n} t / \hbar\right),
$$

where $E_{n}$ is the energy of the compound state, i.e., the sum energy of the pertinent states of the infinitely separated atoms.

In the collisional energy transfer process, the projectile is a straight-line $R(t)=\left(b^{2}+v^{2} t^{2}\right)^{1 / 2}$ with collisional velocity $v$ and impact parameter $b$. The LICET process includes laser field interaction and the multipole collisional interaction, assuming the $\boldsymbol{E}(t)=E_{0} \boldsymbol{a}_{y} \cos (\omega t)$ is the inducing laser field with frequency $\omega$, then the interaction Hamiltonian takes the form:

$$
H(t)=-e y_{A} E_{0} \cos \omega t-e y_{B} E_{0} \cos \omega t+V_{A B},
$$

where the collisional interaction is

$$
V_{A B}=\sum_{j=3} V_{j} R(t)^{-j}
$$

with

$$
V_{j}=\sum_{l=1}^{j-2} \sum_{m=-1<}^{l<} F(j, l, m) Q_{l}^{m}(A) Q_{j-l-1}^{-m}(B) .
$$

Here $l_{<}$is the lesser of $l$ and $j-1-l, Q_{l}^{m}(A)$ and $Q_{j-l-1}^{-m}(B)$ are multipole moment operators, and $F(j, l, m)$ is given by $[19,20]$ :

$$
\begin{aligned}
& F(j, l, m)=(-1)^{j-l-1}\left[\frac{(2 j-2) !}{(2 j-2 l-2) !(2 l) !}\right]^{\frac{1}{2}} \\
& \times C(j-l-1, l, j-1 ; m,-m, 0)= \\
& \quad \frac{(-1)^{j-l-1}(j-1) !}{[(l-m) !(l+m) !(j-1-l-m) !(j-1-l+m) !]^{\frac{1}{2}}},
\end{aligned}
$$

where $C(j-l-1, l, j-1 ; m,-m, 0)$ is the Clebsch-Gordan coefficient.

Then we can obtain the motion equations of the probability amplitudes by using the wave function of the system and the interaction Hamiltonian expressed by Eq. (3):

$$
\text { i } \dot{C}=H C,
$$

where

$$
C=\left[\begin{array}{l}
c_{1} \\
c_{2} \\
c_{3} \\
c_{4}
\end{array}\right]
$$

$$
H=
$$

$\left[\begin{array}{cccc}0 & V_{12} \mathrm{e}^{\mathrm{i} \omega_{12} t} & V_{13} \mathrm{e}^{\mathrm{i}\left(\omega_{13}+\omega\right) t} & V_{14} \mathrm{e}^{\mathrm{i}\left(\omega_{14}+\omega\right) t} \\ V_{21} \mathrm{e}^{\mathrm{i} \omega_{21} t} & 0 & V_{23} \mathrm{e}^{\mathrm{i}\left(\omega_{23}+\omega\right) t} & 0 \\ V_{31} \mathrm{e}^{\mathrm{i}\left(\omega_{31}-\omega\right) t} & V_{32} \mathrm{e}^{\mathrm{i}\left(\omega_{32}-\omega\right) t} & 0 & V_{34} \mathrm{e}^{\mathrm{i} \omega_{34} t} \\ V_{41} \mathrm{e}^{\mathrm{i}\left(\omega_{41}-\omega\right) t} & 0 & V_{43} \mathrm{e}^{\mathrm{i} \omega_{43} t} & 0\end{array}\right]$,

where

$$
V_{m n}=\langle m|H(t)| n\rangle,
$$

and

$$
\omega_{m n}=\left(E_{m}-E_{n}\right) / \hbar .
$$

Using a unitary transformation, $a(t)=T(t) \cdot C(t)$, where

$$
T=\left[\begin{array}{cccc}
\mathrm{e}^{-\mathrm{i} \omega_{21} t} & 0 & 0 & 0 \\
0 & 1 & 0 & 0 \\
0 & 0 & \mathrm{e}^{-\mathrm{i}\left(\omega_{32}-\omega\right) t} & 0 \\
0 & 0 & 0 & \mathrm{e}^{-\mathrm{i}\left(\omega_{42}-\omega\right) t}
\end{array}\right]
$$

Eq. (5) can be simplified as

$$
\mathrm{i} \dot{a}=V a,
$$

where

$$
\begin{aligned}
& a=\left[\begin{array}{l}
a_{1} \\
a_{2} \\
a_{3} \\
a_{4}
\end{array}\right], \\
& V=\left[\begin{array}{cccc}
-\omega_{21} & V_{12} & V_{13} & V_{14} \\
V_{21} & 0 & V_{23} & 0 \\
V_{31} & V_{32} & \omega_{32}-\omega & V_{34} \\
V_{41} & 0 & V_{43} & \omega_{42}-\omega
\end{array}\right], \\
& V_{m n}=V_{n m}^{*} .
\end{aligned}
$$

The final state probability can be obtained by solving motion equations of the probability amplitudes as a function of impact parameter

$$
\sigma=\int_{0}^{\infty}\left|a_{4}(b, t=\infty)\right|^{2} 2 \pi b \mathrm{~d} b .
$$

\section{Results and discussions}

Assuming that the transfer laser field intensity is $10^{6} \mathrm{~V} / \mathrm{m}$ and strictly resonant $\left(\Delta=0 \mathrm{~cm}^{-1}\right)$, the collisional transition probability $\left|a_{4}(t)\right|^{2}$ as a function of time $t$ is calculated for this $\mathrm{Ca}-\mathrm{Sr}$ system (see Fig. 2). The results appear that $\left|a_{4}(t)\right|^{2}$ oscillates over time and strongly depend on the impact parameter $b$. The smaller 
the $b$ is, the stronger the $\left|a_{4}(t)\right|^{2}$ oscillates. The dipoledipole LICET process and dipole-quadrupole $[1,9,10$, $15-17,21]$ are characterized by the collisional time being $\approx 40$ ps. By contrast, we find that, when $|t| \geq 5 \mathrm{ps,}$ $\left|a_{4}(t)\right|^{2}$ approaches to a definite value. It indicates that the laser induced quadrupole-dipole collision process occurs for $\approx 10 \mathrm{ps}$, which is much shorter the collisional time of the dipole-dipole collision process.
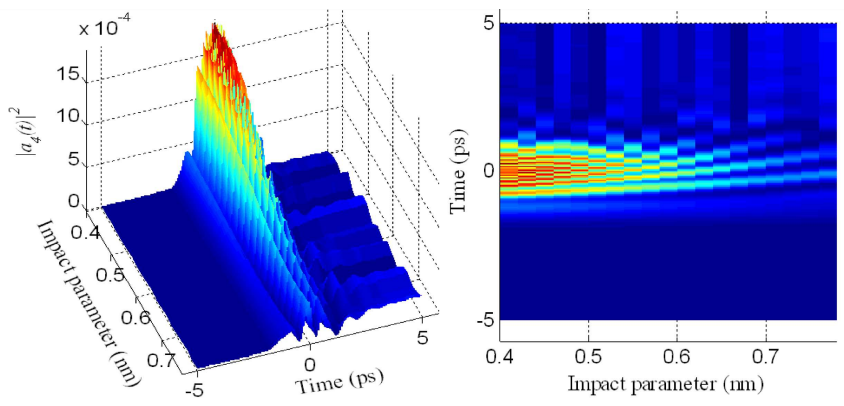

Fig. 2. The time dependence of laser-induced collision transition probability $\left|a_{4}(t)\right|^{2}$ for the Ca-Sr system.

The cross-section as a function of transfer laser detuning in weak field for the Ca-Sr system is shown in Fig. 3. The excitation spectra calculated for the Ca-Sr system show a doublet structure. The magnitude, the FWHM, and the profile of the peak at $\Delta=35.5 \mathrm{~cm}^{-1}$ are obviously different from the one at $\Delta=0 \mathrm{~cm}^{-1}$, indicating that they are corresponding to different mechanisms.

The laser-induced cross-section exhibits a broad maximum at around $\Delta=0 \mathrm{~cm}^{-1}$ indicating that the LICET process is most effective in this region. In weak field (see Fig. 3), the spectra show a universal asymmetric profile which can be seen as a fingerprint for the laser induced excitation transfer process [22] and the asymmetry of the excitation spectra profiles is due to the repulsion interaction of the quasimolecule states. The energy transfer process can be regarded to be a resonant transition process between two compound states as Fig. 4 shows. Because of the repulsion interaction of the quasimolecule states, when $\omega>\omega_{0}$, there are internuclear separations to satisfy the resonant transition condition, which lead to the slow reduce of the cross-section in quasistatic wing. But when $\omega<\omega_{0}$, no such resonance exists for the transition [8].

When the transfer laser is turned at around $\Delta=$ $35.5 \mathrm{~cm}^{-1}$, the applied laser field induces a resonant transition from the ground state to the state of $\operatorname{Sr}\left(7 d^{2}[7 / 2]_{3}\right)$, and then, the quadrupole-dipole collisional coupling between the excited $\mathrm{Ca}\left(4 s 3 d^{1} D_{2}\right)$ and $\operatorname{Sr}\left(5 s 5 p^{1} P_{1}^{0}\right)$ deexcites the $\mathrm{Ca}\left(4 s 3 d^{1} D_{2}\right)$ atom to the ground while it excites the $\operatorname{Sr}\left(5 s 5 p^{1} P_{1}^{0}\right)$ atom to the target state with $\left(5 p^{2}[5 / 2]_{2}\right)$ character. Also, the spectrum at around $\Delta=35.5 \mathrm{~cm}^{-1}$ reflects such a dynamic process. In this process the atom is induced to a real state, while in the LICET process the transfer laser induces the atom to a virtual state. Moreover, although both the laser and collision participate in such a process, it is essentially
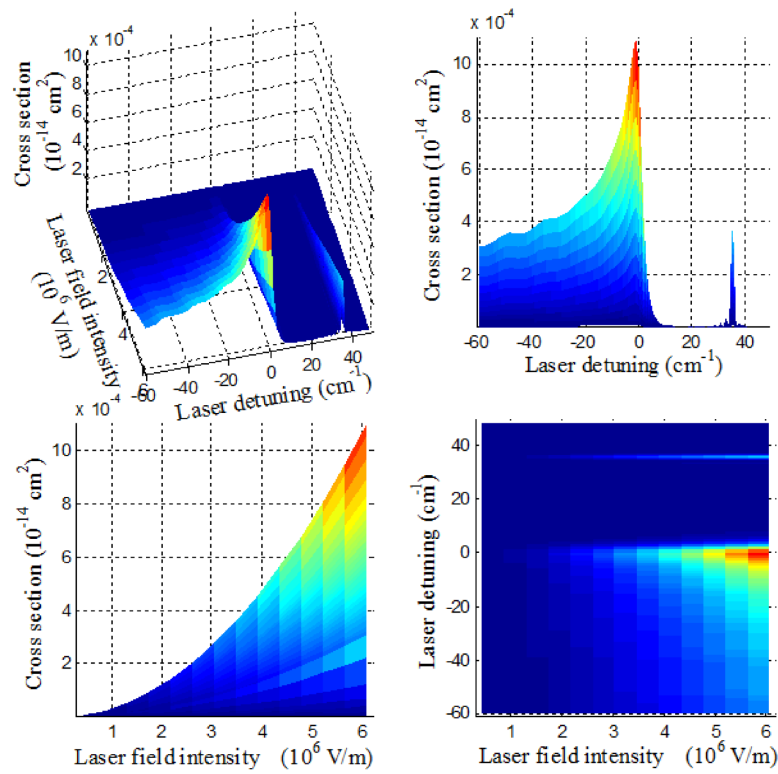

Fig. 3. LICET spectra for $\mathrm{Ca}-\mathrm{Sr}$ system at various transfer laser intensities in weak field.

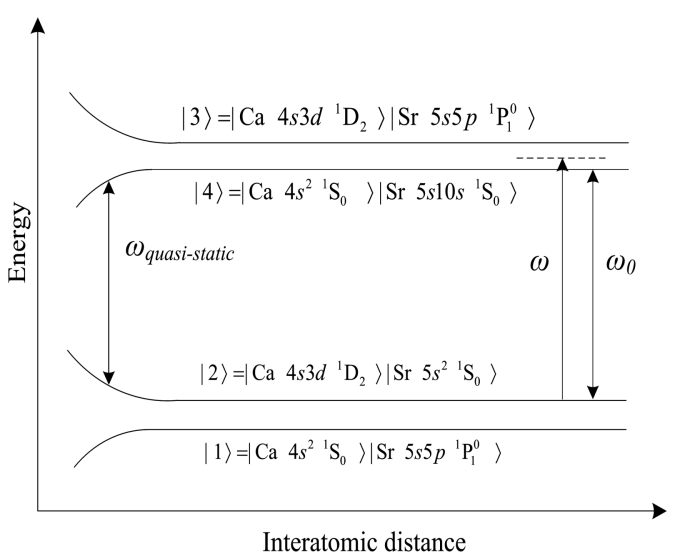

Fig. 4. Schematic adiabatic quasimolecular potentials for the $\mathrm{Ca}-\mathrm{Sr}$ system.

different from the LICET process. Because the actions of the laser and collision are independent but not joint. Therefore the spectrum at around $\Delta=35.5 \mathrm{~cm}^{-1}$ actually ascribes to the collisional energy process and that is why the magnitude, the FWHM, and the profile of the peak at $\Delta=35.5 \mathrm{~cm}^{-1}$ are different from the one at around $\Delta=0 \mathrm{~cm}^{-1}$. But, we note that the spectrum component in the antistatic wing can be used as an effect way to probe the energy level characteristics of the intermediate state, especially for the nonradiative excited intermediate state, i.e., we can study the characteristics of the intermediate state by the radiative characteristics of the final state.

With the increase of the laser field intensity (see Fig. 5), the spectrum at around $\Delta=35.5 \mathrm{~cm}^{-1}$ becomes less obvious comparing with the spectrum at around $\Delta=0 \mathrm{~cm}^{-1}$, thereby indicates that the cross-section of 
interparticle inelastic collisions can be greatly increased by the joint action of the collisional and radiative interactions. Moreover, the resulting spectrum is shifted in frequency toward the antistable wing. An explanation of this is that as the laser intensity increases, the Rabi oscillation frequency in the $\operatorname{Sr}\left(5 s^{2}{ }^{1} S_{0}-5 s 5 p^{1} P_{1}^{0}\right)$ atomic transition increases and become dominant over the Van der Waals quasimolecule mechanism. It tends to enhance the LICET cross-section as $\omega$ approaches to $\omega_{32}$ which is $>\omega_{42}$, resulting in the hypsochromic shift [8]. When the laser field intensity is $1.13 \times 10^{8} \mathrm{~V} / \mathrm{m}$, the maximum occurs at $\Delta=124 \mathrm{~cm}^{-1}$, making this configuration very promising for a quantitative experimental study.
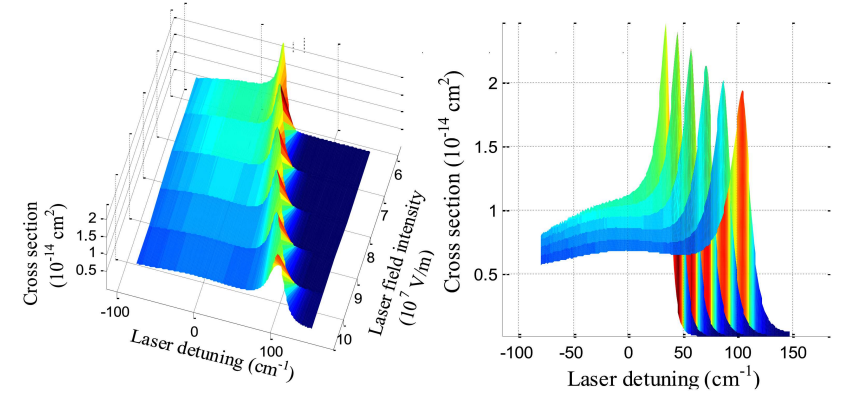

Fig. 5. LICET spectra for the $\mathrm{Ca}-\mathrm{Sr}$ system at various transfer laser intensities in strong field.

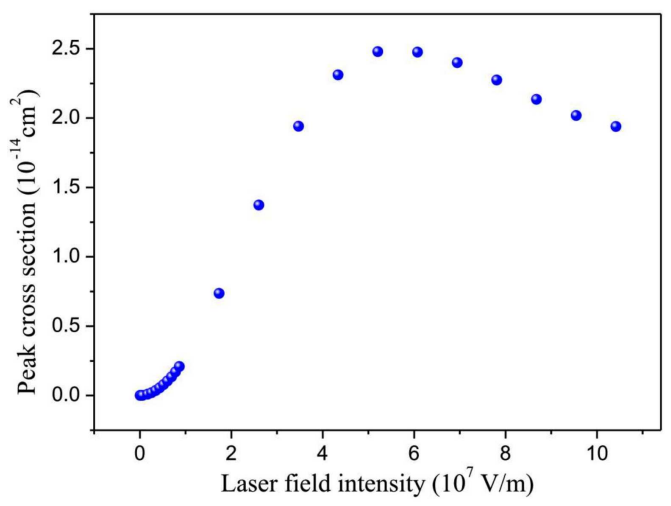

Fig. 6. The peak cross-section as a function of transfer laser intensity for the $\mathrm{Ca}-\mathrm{Sr}$ system.

It has been found that in the previous LICET processes $[10,11,13,21,22]$, the cross-sections in the quasistatic wing are lowered by the presence of a combined light and collisional shift, with the result that the profile becomes more symmetrical at its center. Moreover, the full width at half peak of the profile becomes smaller with the increasing field intensity. It is interesting to observe that in the present system the line shape tends to be more asymmetrical and the quasistatic wing becomes a broad flat which provides us a wide tuning range (see Fig. 5). When the laser field intensity increases from $2.6 \times 10^{7} \mathrm{~V} / \mathrm{m}$ to $8.7 \times 10^{7} \mathrm{~V} / \mathrm{m}$, the FWHM increases from $18.4 \mathrm{~cm}^{-1}$ to $24.3 \mathrm{~cm}^{-1}$. All the variation characteristics are ascribed to the increase of the effective time for level crossing caused by the combined light and collisional shift [21].

The peak cross-section as a function of transfer laser intensity for the $\mathrm{Ca}-\mathrm{Sr}$ system is given in Fig. 6. The collision cross-section rises with laser intensity and tends to saturate. There is a maximum in our results. A crosssection of $2.48 \times 10^{-14} \mathrm{~cm}^{2}$ at a laser field intensity of $5.21 \times 10^{7} \mathrm{~V} / \mathrm{m}$ is obtained which indicates that the laser induced quadrupole-dipole and dipole-dipole collision have a comparable magnitude. The quadrupoledipole collisional process still can be an effective way to transfer energy selectively.

\section{Conclusion}

A laser induced quadrupole-dipole collisional energy transfer system of $\mathrm{Ca}-\mathrm{Sr}$ in which both species made parity nonallowed transitions is proposed, and a four-state model for the laser induced quadrupole-dipole collisional energy transfer process is presented. The results show that the quadrupole-dipole LICET process is much faster than the dipole-dipole LICET process. The excitation spectrum calculated for the $\mathrm{Ca}-\mathrm{Sr}$ system shows a doublet structure, which has never been reported in previous works [1-18]. The two peaks are corresponding to the LICET process and the collisional energy transfer process, respectively. The peak in the antistatic wing can be used as an effect way to probe the energy level characteristics of the intermediate state, especially for the nonradiative excited intermediate state, i.e., we can study the characteristics of the intermediate state by the radiative characteristics of the final state. By contrast, our resulting LICET spectrum is about twice wider than the calculation results in the dipole-dipole, dipole-quadrupole and quadrupole-quadrupole LICET systems $[1,9,10,15$, $17,22]$. It has been interesting to observe that with the increasing field intensity the full width at half peak of the profile becomes larger and the quasistatic wing becomes a broad flat, which are opposite to the results obtained from dipole-dipole, dipole-quadrupole and quadrupolequadrupole LICET process. Such a new variation characteristic indicates that the compound system energy level shift in the present system caused by joint action of the laser field and collision interactions is an important complement to the previous reported type. A crosssection of $2.48 \times 10^{-14} \mathrm{~cm}^{2}$ at a laser field intensity of $5.21 \times 10^{7} \mathrm{~V} / \mathrm{m}$ is obtained which indicates that the quadrupole-dipole process also can be the effective way to transfer energy selectively.

\section{Acknowledgments}

This work is supported by the National Natural Science Foundation of China (Grant No. 11304240) and the Fundamental Research Funds for the Central Universities (Grant No. JB140511). 


\section{References}

[1] R.W. Falcone, W.R. Green, J.C. White, J.F. Young, S.E. Harris, Phys. Rev. A 15, 1333 (1977).

[2] A. Débarre, J. Phys. B At. Mol. Phys. 16, 431 (1983).

[3] C. Brechignac, P. Cahuzac, P.E. Toschek, Phys. Rev. A 21, 1969 (1980).

[4] F. Dorsch, S. Geltman, P.E. Toschek, Phys. Rev. A 37, 2441 (1988).

[5] B. Cheron, H. Lemery, Opt. Commun. 42, 109 (1982).

[6] W.R. Garrett, S.D. Henderson, M.G. Payne, J. Opt. Soc. Am. B 4, 133 (1987).

[7] M. Matera, M. Mazzoni, R. Buffa, S. Cavalieri, E. Arimondo, Phys. Rev. A 36, 1471 (1987).

[8] S. Geltman, Phys. Rev. A 45, 4792 (1992).

[9] S. Harris, J. White, IEEE J. Quantum Electron. 13, 972 (1977).

[10] A. Bambini, P.R. Berman, Phys. Rev. A 35, 3753 (1987).

[11] A. Agresti, P.R. Berman, A. Bambini, A. Stefanel, Phys. Rev. A 38, 2259 (1988).
[12] L.I. Gudzenko, S.I. Yakovlenko, Sov. Phys.-JETP 35, 877 (1972)

[13] A. Bambini, S. Geltman, Phys. Rev. A 50, 5081 (1994).

[14] D.Y. Chen, Q. Wang, Z.G. Ma, Acta Opt. Sin. 16 , 1653 (1996).

[15] D.Y. Chen, Q. Wang, Z.G. Ma, Sci. China Ser. A 27, 449 (1997).

[16] H.Y. Zhang, D.Y. Chen, Z.Z. Lu, R.W. Fan, Y.Q. Xia, Acta Phys. Sin. 57, 7600 (2008).

[17] W.R. Green, M.D. Wright, J. Lukasik, J.F. Young, S.E. Harris, Opt. Lett. 4, 265 (1979).

[18] J.C. White, Opt. Lett. 5, 239 (1980).

[19] J.O. Hirschfelder, W.J. Meath, Adv. Chem. Phys. 12, 3 (1967).

[20] M.E. Rose, J. Math. Phys. 37, 215 (1958).

[21] A. Bambini, M. Matera, A. Agresti, M. Bianconi, Phys. Rev. A 42, 6629 (1990).

[22] Z.Z. Lu, D.Y. Chen, R.W. Fan, Y.Q. Xia, Phys. Rev. A 85, 063402 (2012). 